Théologiques

Théologiques

\title{
Brève histoire de la caricature des figures majeures du christianisme
}

\section{François Boespflug}

Volume 17, numéro 2, 2009

Querelles d'images?

URI : https://id.erudit.org/iderudit/044064ar

DOI : https://doi.org/10.7202/044064ar

Aller au sommaire du numéro

\section{Éditeur(s)}

Faculté de théologie et de sciences des religions, Université de Montréal

\section{ISSN}

1188-7109 (imprimé)

1492-1413 (numérique)

Découvrir la revue

\section{Citer cet article}

Boespflug, F. (2009). Brève histoire de la caricature des figures majeures du christianisme. Théologiques, 17(2), 85-110. https://doi.org/10.7202/044064ar
Résumé de l'article

L'affaire des caricatures de Mahomet (février 2006) a mis en lumière l'existence de seuils de tolérance à la moquerie visant les choses religieuses. Ces seuils varient beaucoup d'une religion à une autre. L'auteur entend souligner à quel point ils évoluent aussi à l'intérieur de la religion chrétienne, réputée plus placide que d'autres à cet égard. Avec une méthodologie d'historien et des repères de théologiens, il retrace les cinq grandes étapes (ou situations typiques) d'une histoire spécifique, celle des rapports entre le christianisme et la dérision, notamment sous la forme graphique de la caricature des figures caractéristiques de cette religion : l'Antiquité, le Moyen Âge, la Réforme, les dernières décennies $\mathrm{du} \mathrm{XIX}^{\mathrm{e}}$ siècle, puis la seconde moitié $\mathrm{du} \mathrm{xx}^{\mathrm{e}}$ siècle. Il ressort que les seuils de tolérance ne sont jamais fixés une fois pour toutes et surtout varient selon qu'il s'agit de Dieu (du Christ) et de la Vierge, ou bien des saints, des curés ou des pratiques religieuses. En tout état de cause, ils sont toujours l'objet de subtiles négociations et font partie intégrante du pacte social dont chaque société a besoin pour éviter les heurts qui peuvent résulter du choc des sensibilités. 


\title{
Brève histoire de la caricature des figures majeures du christianisme
}

\author{
François BCESPFLUG" \\ Histoire des religions \\ Université de Strasbourg, France
}

L'affaire des caricatures du Prophète Mahomet qui a éclaté en février 2006 a été et demeure une occasion de s'interroger, entre autres, sur les rapports entre les religions et la dérision, notamment sous la forme graphique de la caricature des principales figures caractéristiques de chaque religion. Or, il semblerait que parmi les croyants, et même parmi les observateurs censément avisés qui ont écrit à ce sujet, l'on n'ait pas toujours une conscience très claire de l'historicité intrinsèque des seuils de tolérance de la moquerie appliquée aux choses religieuses. Ces seuils ne sont jamais fixés une fois pour toutes et se déplacent selon les époques, les lieux et les courants. Ils varient aussi, beaucoup plus que l'on ne le croirait, selon les sujets de moquerie. Et en tout état de cause, ils sont toujours l'objet de subtiles négociations et font partie intégrante du pacte social dont chaque société a besoin pour éviter les heurts qui peuvent résulter du choc des sensibilités.

C'est ce que je voudrais illustrer dans ce qui suit, à la lumière du cas chrétien. Après avoir pris part au débat sur le fond de l'affaire par la publication d'un livre paru dès avril 2006 (Bœespflug 2006a1), il me paraît en

* François Bœspflug, dominicain, est professeur d'histoire des religions à la Faculté de théologie catholique de l'Université de Strasbourg. Spécialiste d'iconographie chrétienne depuis sa thèse sur Dieu dans l'art (1984), il enseigne aussi au Centre Sèvres (Facultés jésuites de Paris) et a été le titulaire de la Chaire du Louvre 2010. Parmi ses dernières publications, on trouve (2008) Dieu et ses images. Une histoire de l'Éternel dans l'art, Paris, Bayard et (2010) Le Dieu des peintres et des sculpteurs. L'Invisible incarné, Paris, Hazan/Musée du Louvre Éditions.

1. Voir aussi Bœspflug (2008). Pour une analyse du savant montage politique de cette affaire, voir Sifaoui (2006). 
effet utile de revenir sur les rapports entre le christianisme et la dérision en tentant de repérer les étapes d'une histoire spécifique, celle de la «moquerie-en-images» frappant les figures qui sont traditionnellement considérées comme les insignes distinctifs de la foi chrétienne. À quand remontent les premières «agressions » de ce genre? Quelles furent, parmi les images chrétiennes, les premières "victimes" ? Certaines figures ont-elles, au moins jusqu'à une certaine date récente, été préservées de toute atteinte? Depuis quand est-il toléré que les artistes se moquent, pêle-mêle, "des choses de la religion» (de Dieu, de la Bible, du clergé, du catéchisme, etc.) en caricaturant ses figures traditionnelles? Après enquête, il me semble que l'on peut distinguer cinq situations-clés dans l'histoire de la dérision appliquée aux figures les plus sacrées du christianisme. Ce sont l'Antiquité, le Moyen Âge, la Réforme, les dernières décennies du XIX ${ }^{\mathrm{e}}$ siècle puis la seconde moitié du $\mathrm{XX}^{\mathrm{e}}$ siècle.

\section{Dans l'Antiquité}

Abstraction faite d'intailles où le motif du Crucifié nimbé apparaît pour la première fois mais sans la croix, les premières représentations du Christ en croix, à savoir deux reliefs, l'un en bois, sur un panneau de la porte de Sainte-Sabine à Rome, et l'autre en ivoire, conservé à Londres, ne sont pas antérieures au début du ve siècle (Skubiszewski 2002). Or, l'on connaît depuis sa découverte en 1856 une caricature du Christ en croix datant du début du III siècle. Il s'agit d'un graffito gravé au couteau ou avec la pointe d'un clou montrant, à la place du Christ en croix, un homme à tête d'âne, représenté de dos les fesses dénudées, tandis qu'un homme se tient debout sur la gauche, avec pour légende Alexamenos sebete theon ("Alexamenos adore $\left.\operatorname{Dieu}^{2} »\right)$ - une autre inscription assurant que cet Alexamenos est un fidelis, un chrétien. Si tel est bien le cas, c'est à coup sûr l'indice d'une haine de ce chrétien en particulier ou des chrétiens en général, du temps où ceux-ci étaient perçus comme des parias, des athées, des gens dangereux pour le pacte social et la sécurité de l'État. On leur aurait donc adressé le grief d'"onolâtrie » (ils adorent un âne, ou un homme onocéphale, à tête

2. Dessin gravé du Paedagogicum (Antiquarium del Palatino) conservé au musée des Thermes à Rome; voir Dinkler (1967), Skubiszewski (2002, 15), Heid (2006, 1102). 
d'âne), grief qui avait été d'abord adressé aux juifs (Leclercq 1907, col. 2041-20423).

Cette caricature est un hapax. D'autres formes de moquerie ont visé le christianisme naissant. La dérision inaugurée par Celse au II $^{\mathrm{e}}$ siècle dans son Contre les chrétiens, auquel répondit le Contre Celse d'Origène, s'est poursuivie, mais uniquement sous forme littéraire: songeons à l'antichristianisme déclaré du Misopogon, un écrit satirique de Julien l'Apostat (332363), lequel était convaincu que le triomphe du christianisme avait précipité le déclin de l'Empire et qu'il était donc temps de restaurer les cultes païens ${ }^{4}$. Quoi qu'il en soit, durant des siècles, semble-t-il, la dérision antichrétienne ne se manifesta plus sous forme plastique. Une fois Constantin au pouvoir et $a$ fortiori à partir du règne de Théodose (fin du IV siècle), les images de Dieu en majesté puis celles du Pantocrator, de la Vierge à l'Enfant et du Christ crucifié vont être constituées en archi-symboles du christianisme. Et durant tout le temps de la civilisation chrétienne, jamais elles ne furent l'objet de moqueries, de caricatures ou de vandalisme avéré, sauf exceptions qui se comptent sur les doigts de la main. Il ne faut pas prendre pour argent comptant les rumeurs qui attribuèrent aux juifs, dès l'Antiquité, des intentions profanatrices.

On mentionne [...] qu'au temps de Théophile, le patriarche d'Alexandrie [384-412], les juifs fabriquèrent une croix en bois et $y$ dessinèrent une forme humaine, afin de pouvoir transpercer [cette image] par dérision. Quand on la perça, il en sortit du sang et de l'eau, au point qu'ils coulaient à terre. Cela se passa le quatorze du mois de Misrâ, jour qui fut enregistré parmi les commémoraisons dans les livres officiels de l'Église ${ }^{5}$.

3. «Apion [Histoire de l'Égypte], le grammairien d'Alexandre, répandit dès le premier siècle de notre ère la fable que le roi d'Assyrie Antiochus-Épiphane, entrant à main armée trois siècles plus tôt dans le temple de Jérusalem, y vit en place d'honneur une tête d'âne en or. Plutarque [Symp., IV, 5] et Tacite [Historia, V] se sont faits les échos de cette assertion, et comme les païens de Rome confondaient dans un même ensemble juifs, gnostiques et chrétiens, ce furent ces derniers surtout qui furent accusés d'asinolâtrie ", Charbonneau-Lassay $(2006,225)$. Tertullien dénonce à plusieurs reprises des images contemporaines portant l'inscription Deus christianorum onochoétès [de race d'âne] (Apologétique, XVI; Ad Nationes, XIV).

4. En 1909, «le conseil municipal de Paris aurait rêvé de dresser une statue en l'honneur de Julien l'Apostat qui eût ainsi remporté une retentissante victoire posthume propre à effacer sa célèbre parole: “Tu as vaincu, Galiléen!” ” Cité par J. Lalouette (1992, 52).

5. Lampe des ténèbres, d'Abu-I-Barakat ibn Kabar, prêtre de l'église Al-Moallaqa au Vieux-Caire, mort en 1324; cité dans Zanetti (1991, 84-85). 
Ce témoignage correspond trop bien aux intérêts du christianisme médiéval pour ne pas apparaître à la fois très tardif et très suspect.

\section{La période médiévale}

Les médiévaux se délectaient du grotesque, du monstrueux, de l'hybride et des drôleries en tous genres (Baridon, M. Guédron 2006, 12). Ils excellaient dans le paradoxe (Dandrey 1997), le rire (Horowitz et Menache 1994), le double sens et l'équivoque (Roy 1992). Dans l'art médiéval, la moquerie fut parfois poussée très loin, par exemple contre les clercs, mais elle ne se risqua pas, sauf exceptions, du côté des images les plus sacrées, celles de Dieu, du Christ en majesté ou en Croix, ni de Marie. "L'humour parodique a connu au Moyen Âge une faveur exceptionnelle ${ }^{6}$ ". Mais il fut exercé pour l'essentiel sur des textes - encore faut-il préciser: des textes latins. On connaît ainsi plusieurs parodies de la messe et toutes sortes de sotties (Strubel, «Sottie », DMA, 1348-13497), de «sermons joyeux " (sur "saint Hareng» ou "sainte Andouille ${ }^{8}$ ») et d'amusements sur les prières usuelles, tel l'Office de l'âne, qui ont été recueillis dans les Carmina burana, recueil satirique de poésies latines ${ }^{9}$ rassemblées en Allemagne au XIII ${ }^{\mathrm{e}}$ siècle, sans parler de textes burlesques comme le Testamentum asini, un texte

6. Roy («Parodie», DMA, 1049): «On peut se demander quelle part réelle de subversion comportait cette littérature [...]. Tout indique que la subversion n’y ait été présente qu'à l'état minimal. En effet, dans le tissu serré de l'axiomatique médiévale, l'inversion parodique des valeurs n'était au fond qu'une occasion de communier avec les contemporains, le temps d'un clin d'œil, en prenant prétexte des institutions les mieux connues et les plus respectées. »

7. Lehmann a édité des textes de messes ludiques (Spielmessen), d'après des manuscrits du XIII et du XIV ${ }^{\mathrm{e}}$ siècles.

8. Voir Strubel («Sermon joyeux ", DMA, 1329) et aussi Delumeau (1981).

9. Roy ( Satire et burlesque », $D M A, 1283-1284)$ : la satire gagne aussi le genre narratif avec le Speculum stultorum de Nigel Wireker (1140-1200, qui raconte comment l'âne Burnellus, figure du moine cistercien, à la recherche d'une queue plus longue, ne trouve rien de mieux que de la perdre...), et contamine encore le domaine des contes folkloriques d'animaux comme Ysengrin (le loup, incarnation d'un moine élevé à l'épiscopat, très souvent humilié par le goupil Renart, et qui finit dévoré par les porcs). Voir à ce sujet Tilliette («Ysengrinus», DMA, 1483). Mais ces dénonciations des désordres sont au fond une exaltation en creux de l'ordre. La conclusion de cet article diffère de celle de l'article "parodie» du même auteur: «l'enjeu était en réalité considérable, en ce qu'il menait à une déconstruction de toutes les valeurs: le sacré s'y trouvait mis à l'épreuve du trivial, le sapientiel à celle du banal, et le truisme cédait la place à l'absurde. En ce sens, le burlesque fut sans doute plus corrosif pour les institutions que ne le fut la satire lettrée. » 
dans lequel un âne destine ses oreilles aux cardinaux, sa queue aux frères mineurs, etc. Les occasions de défoulement ne manquaient pas - en témoignent les charivaris, carnavals et autres fêtes des fous, inversion burlesque des institutions en place, véritable triomphe rituel de l'indiscipline et de l'insolence (Heers 1983). Mais l'on y gardait le sens exact des bornes à ne pas dépasser, et tout rentrait dans l'ordre dès le lendemain.

Se moquer de Dieu et défigurer ses signes, l'on s'en gardait bien, car c'était aller au devant de graves sanctions et se mettre ipso facto au ban de la société chrétienne. Au XIII siècle, en effet, s'instaure, dans les pays de chrétienté, un système répressif punissant toute atteinte à l'honneur dû au nom de Dieu, depuis le simple juron jusqu'au blasphème intentionnel en passant par le parjure - les péchés de la langue sont l'objet de toute l'acribie du moraliste (Casagrande et Vecchio 1991; Delaurenti 2007) et de la surveillance sourcilleuse du pouvoir politique. Le «bon roi » Louis IX, alias saint Louis, prône une justice sur ce point comme sur d'autres beaucoup plus rigoureuse que la justice épiscopale, tenue pour laxiste, ou que celle du Saint-Siège lui-même, défavorable à toute mutilation corporelle en cas de blasphème. Le mal-parler de Dieu, en «doulce France», devient alors un crime d'État. On raconte que saint Louis ne tolérait aucun juron pendant les traversées en mer. Même celui qu'on laissait échapper au marché dans un moment de colère fut réprimé avec une rigueur exceptionnelle. Car la prononciation fautive du nom de Dieu était vécue comme une menace pesant sur toute la société. Offensé, Dieu allait se venger, tôt ou tard, en déchaînant la série noire: guerres, séditions, famines et pestilences. Le respect du bien dire et la répression du mal-dire (du maudire) étaient donc affaire de salut public. Le Code pénal promulgué par saint Louis était sans doute impossible à appliquer à la lettre, mais il stipulait, au-delà de la deuxième ou de la troisième récidive avérée, que punissaient de "simples amendes» (au demeurant fort lourdes), de marquer le nez au fer chaud, de trouer voire de trancher la langue du coupable, puis de lui fendre la lèvre inférieure, etc. La répression du blasphème n'était pas plus tendre dans l'Espagne d'Alphonse X, roi de Castille et de Léon ${ }^{10}$.

Durant les trois derniers siècles du Moyen Âge, la dose de jeu, d'ironie et de moquerie qui affleure dans les images varie selon les lieux et les temps.

10. À ceci près que nobles et vilains échappaient aux peines corporelles, qui ne frappaient par conséquent que les serfs. "La peine la plus grave (les mains coupées) est réservée au blasphème par action, par exemple quand quelqu'un a craché sur une croix ou l'a foulée aux pieds» (F. LECLER, «Blasphème », Catholicisme, t.2, col. 78-83 [82]). 
Certaines formes d'art ont été des nids à moquerie, telles les frises sculptées de façades d'église - où l'on découvre parfois des singeries du culte voire de la messe -, ou les marges des livres d'heures et autres manuscrits à peinture, avec leurs drôleries, qui semblent se multiplier à partir de la seconde moitié du XIII siècle et parviennent entre 1250 et 1350 à la consistance d'un genre à part entière ${ }^{11}$. Ces singeries et autres insolences frisent le blasphème, mais savent se retenir d'aller trop $\operatorname{loin}^{12}$. La moquerie directe s'en prend parfois aux symboles les plus sacrés, par exemple quand est peint un singe déféquant dans un calice (Wirth 2000, 442-444), mais elle épargne les figures divines, sauf de manière indirecte, en représentant ceux qui les agressent.

Dans la Bible moralisée dite de Tolède (vers 1230), manuscrit riche d'environ 5000 médaillons peints et confectionné à Paris pour l'éducation politique et religieuse du jeune Louis IX, futur saint Louis, les juifs sont souvent maltraités, voire caricaturés (on les représente alors de profil, avec barbe et nez crochu); les musulmans, à l'inverse, sont épargnés - ils brillent même par leur absence (y compris dans les textes de moralisation, qui ne les évoque qu'une quinzaine de fois, alors qu'ils mentionnent les juifs à tout propos $\left.{ }^{13}\right)$ : c'est surprenant, dans un manuscrit prodigue en audaces et datant de l'époque des croisades. La moquerie vise avant tout les clercs de toute bure et de tout rang, qui apparaissent parfois en simoniaques ou en débauchés, recevant des pots-de-vin, jouant dans des tavernes ou enlaçant une femme au lit, fussent-ils évêques ${ }^{14}$. On y trouve également une figure de juif se détournant de Dieu ou du Crucifix et/ou lui tirant la

11. Sur les drôleries, voir Randall (1966), Camille (1992), Wirth (2000), Mellinkoff (2004) et Wirth et Engammare (2008).

12. Dans une miniature conservée à New York (Une initiale ornée $G$, par Stefano da Verona, New York, PML, M 558 D), les trois Personnes d'une Trinité à l'autel sont figurées en vieillards assis à une table à tréteaux vue en perspective, chacune étant placée devant un calice surmonté d'une patène; elles bénissent et tiennent chacune un livre; un lapin joue au premier plan, un cerf court à l'arrière-plan et un âne broute dans un paysage de collines: honni soit qui mal y pense (Arte Veneta, 57, 2000, p. 11 et fig. 19 p. 20).

13. Caroff (2006) relève que «les musulmans ont moins subi l'outrage de la caricature que d'autres "étrangers" qui ont été jugés à l'époque médiévale beaucoup plus dangereux, comme les juifs, mis au rang de suppôts du diable ou de déicides, ou considérés comme plus différents ou lointains, comme les Africains» (p. 183); et l'auteur de renvoyer à Sansy (1994), Mellinkoff (1993), Devisse, et al. (1979). De toute façon, les musulmans n'ont guère servi à figurer «l'altérité » au Moyen Âge, ou du moins pas avant la fin du XIV ${ }^{\mathrm{e}}$ siècle.

14. Ainsi dans la Bible moralisée de Tolède, t. 2, f. 141 v, d. 
langue ${ }^{15}$. C'est une première, à ma connaissance, dans l'histoire de l'art médiéval. Elle insinue que les juifs, non contents d'être un peuple déicide, ajoutent à leur crime celui de blasphème, «en anathématisant le nom chrétien trois fois par jour dans toutes les synagogues ${ }^{16} »$. Mais la Bible moralisée du XIII ${ }^{\text {e }}$ siècle est un document d'une inventivité exceptionnelle, qui ne fera jamais école, du moins sur ce terrain précis.

\section{Des Réformes à la fin de l'Ancien Régime}

À l'époque des Réformes, la tradition déjà bien établie de moquerie à l'égard du clergé est portée à son comble. La gravure, monochrome ou polychrome, devient une arme de combat. Elle est beaucoup utilisée par les protestants pour diffuser leurs idées. D'où les caricatures anti-monastiques ou antipapistes ${ }^{17}$, telle la feuille volante intitulée «la cornemuse du diable», xylogravure coloriée montrant le diable jouant à travers la tête d'un moine

15. Même quand il ne tire pas la langue, le blasphémateur, bête ou homme, juif ou non, a en général la bouche ouverte. Voir Tol, I, 71 v, b (sur Nb 14,1-4) et III, f. 59, a, 64 D (le mauvais larron), 66A, $179 \mathrm{v}, \mathrm{B}$ et $185 \mathrm{v}$, D. Je ne connais pas de médaillon où un homme ferait la figue à Dieu, même dans le contexte de la Passion. Ce geste-là n'apparaît, me semble-t-il, que dans la panoplie des arma Christi, plus tard. La figure la plus audacieuse de juif tirant la langue à Dieu (Dieu au ciel ou Dieu-homme en croix) est en Tol I, f. 58, D et d, sur Lv 24, 10-11 (dans Laborde 1912-1927, I, pl. 64, médaillons $\mathrm{D}$ et $\mathrm{d}$, un juif caricaturé par son visage passablement bestial, se moque de Dieu, semble-t-il, mais sans lui tirer la langue). La suite (f. 59, a et B), qui traite du même sujet, ne donne pas lieu à reprise de la figure du blasphémateur en train de blasphémer.

16. Tol, II, f. 104, c, en moralisation d'Is 5,18 (Ve qui trahitis iniquitatem in funiculis uanitatis et quasi uinculum plaustri peccatum), en face d'un texte qui accuse les chefs des juifs de blasphème (Hec dicuntur ad principes iudeorum qui sepe ad penitentiam vocati usque hodie perseuerant addentes peccata peccatis contumaciam scilicet et blasphemiam homicidio qui ter per singulos dies in omnibus synagogis anathematizant uocabulum christianum). La langue tirée du juif de gauche est beaucoup plus nettement tracée dans Laborde (1912-1927, t. II, pl. 328).

17. Jusqu'à traîner dans la boue les figures du pape et de l'Église sous forme allégorique, parfois associées l'une et l'autre à une troisième qui n'est pas en meilleure posture, celle du musulman (du Turc) : ainsi dans une xylogravure luthérienne du XVI siècle qui montre le Christ ressuscité piétinant une bête de l'apocalypse figurée sous la forme d'une hydre tricéphale, avec une tête de pape tiaré, une tête d'Église et une tête de Turc (Webster 1990, 80). Voir, entre tant d'autres, dans Muller (1997), les cat. $n^{\circ} 79$ (allégorie de l'ancien et du nouveau dieu), $n^{\circ} 95$ (les ecclésiastiques nécrophages) et $n^{\circ} 97$ (pape et ecclésiastiques en loups attrapant des oies). 
dont le nez se prolonge en flûte avec ses trous ${ }^{18}$, ou la double tête du pape et du diable, ou la gravure de Henri Vogtherr l'Ancien représentant le pape en antéchrist (Zurich, 1546; voir Hommeanimal, 2004, n IV, p. 41; voir aussi Muller 2000). L'ouvrage de Luther intitulé La papauté romaine établie par Satan comporte en frontispice une gravure montrant le pape pourvu d'oreilles d'âne et chevauchant un porc ${ }^{19}$. Le pape fut aussi figuré en âne - le bestiaire des anticléricaux est varié, et il a toute une histoire, et la gravure protestante promeut le porc plutôt que l'âne ${ }^{20}$. Les catholiques, il va sans dire, rendirent aux protestants la monnaie de leur pièce ne se gênant pas pour diffuser par exemple des images d'un Luther à sept têtes, en Bête de l'Apocalypse.

La fréquentation de ce corpus des insultes graphiques à visée polémique, difficile à dominer tant il est vaste, irait néanmoins à établir que dans les gravures, de quelque bord qu'elles fussent, l'on ne s'en prend ni à la figure de Dieu ni à celle du Christ ${ }^{21}$ ou de sa mère: ce n'est pas après eux que l'on en a, mais après les ministres ou représentants des deux camps. S’il arrive que l'on nourrisse des pensées gaillardes à l'encontre du Tout Puissant, de son divin Fils ou de leur commun Esprit Saint, du moins les garde-t-on pour soi et ne prend-on pas le risque de les exprimer en clair ni

18. Maître allemand inconnu, vers 1530-1535 (dimension de la feuille: 364 x $270 \mathrm{~mm}$ ): "caricature des ordres monastiques dont les péchés réels ou imaginaires ont fait à l'époque de la Réforme l'objet de diverses représentations assez crues » (Schäfer, dans Gravures du temps de la Réforme, 2004, cat. $\mathrm{n}^{\circ} 35$, p. 39).

19. Cela est relevé par Molanus, Traité des saintes images, 1. 2, chap. 70 (éd. Bœespflug et al., t. 1, p. 332). Voir la gravure sur bois intitulée «Pape et ecclésiastiques en conversation avec le diable", de 1520, dans Muller (1997), cat. $n^{\circ}$ 65, puis 70 et 72 (le diable étant remplacé par Luther puis saint Paul).

20. Lalouette $(1992,56)$ : au XIX siècle, il comprendra «le corbeau, le cochon, qui arrivent en tête, le hibou, l'araignée, le serpent, le rat, le bouc, le pou, un peu moins souvent, le singe, l'âne, l'oie ", etc. Voir sa note 22 sur le bestiaire du temps de la Réforme.

21. Molanus, Traité des saintes images, 1. II, chap. 70 (éd. citée, p. 332-333), rapporte cependant: "J'ai vu moi-même les effigies des larrons que l'on peint d'habitude autour de l'image du Christ entières et intactes, tandis que la figure de Jésus-Christ était défigurée, lacérée et déchirée par les poignards, les épées et les lances, et celle qui décrivait l'archange saint Michel mise en pièce alors que celle du démon malfaisant demeurait entière et intacte. » Il se pourrait cependant que ce témoignage soi-disant oculaire n'ait pas la moindre valeur historique, sauf à lui reconnaître celle d'un argument polémique. Certaines actions iconoclastes étudiées par Olivier Christin (1991) révèlent en tout cas une sélectivité opposée à celle qu'évoque Molanus, les détériorations ne touchant que les représentations du clergé, des saints ou des donateurs, et épargnant les personnes divines, notamment le Christ. 
de les dessiner sous la forme de caricatures peintes ou gravées (Dinzelbacher 1999; Minois 1998, chap. 3). On a soutenu qu'un étrange dessin de Grünewald, vers 1520, pourrait représenter une Anti-trinité diabolique (Baltrusaitis 1981, 41; Vaisse 1974, cat. 74, p. 101). Mais cette interprétation est discutée et paraît improbable.

Les images qui auraient pu fournir l'occasion d'une atteinte à la figure du Christ, comme les miniatures médiévales mettant en scène la figure du fou et illustrant le psaume 14 (13),1 (Dixit insipiens in corde suo: non est Deus), sont en fait des images au second degré, pour dénoncer ceux qui méprisent $\mathrm{Dieu}^{22}$ et commettent des sacrilèges ${ }^{23}$. Elles paraissent aujourd'hui timides, comparées aux actuelles caricatures antichrétiennes ou antijuives. Une des rares images du XVI ${ }^{e}$ siècle où l'on se moque du Christ hors du cycle de la Passion - et donc sans la bonne excuse des bourreaux du prétoire - montre un homme qui lui tire les poils de la barbe (Bartsch 1920-1923, t. 10, 4 [117], p. 99, "The Mocking of Christ»). Mais cette gravure qui se trouve dans la Grande Passion de Dürer a été conçue pour dénoncer les blasphémateurs, non pour faire chorus avec eux ou se cacher derrière eux: l'histoire du blasphème dans l'art a donc évité tout blasphème en image pour se restreindre à la figure du blasphémateur en train d'attenter au Christ ou à l'image du Christ ${ }^{24}$.

Le corps de lois réprimant le blasphème fut en vigueur jusqu'à la fin de l'Ancien Régime et resta sévère dans ses applications. La perception médiévale d'une nécessaire retenue de la langue, s'agissant de la prononciation du vocable Dieu, s'est longtemps maintenue. Après un ou deux siècles plus portés à la mansuétude, la tendance au retour à une rigueur parfois accablante, dans l'Europe moderne, s'affirme un peu partout (Christin, 1994), y compris à Rome. Dans l'Empire et les pays germaniques, l'on va jusqu'à

22. Un des exemples les plus «osés» que je connaisse est offert par une miniature napolitaine montrant le fou renversé en arrière et paraissant vouloir frapper Dieu: Vienne, ÖNB, ms. 1921, f; 65 v, repr. dans Meiss (1969, fig. 416).

23. Gravure d'Anton Koberger, "Un sacrilège pique sa lance dans le flanc du Christ ", 1493 ; repr. dans Clair $(2005,142)$.

24. De même, certains iconoclastes ont pu se montrer sélectifs et viser spécialement les figures de Dieu, du Christ ou de la Vierge, en sorte de commettre un vandalisme blasphématoire voulu comme tel. Longtemps le peintre avait été assimilé à un blasphémateur. La situation entre-temps s'était retournée, pour en revenir à ce qu'il en était du temps de la Querelle des images. C'est désormais, ou de nouveau, l'iconoclaste qui est assimilé à un blasphémateur - en milieu catholique romain, s'entend. Mais c'est exceptionnel à cette époque. 
la peine de mort. Le sommet de la lutte contre les blasphémateurs est atteint au XVI ${ }^{\mathrm{e}}$ siècle. Il coïncide avec le combat contre les hérétiques, les sorcières, les juifs et les Turcs: le monde chrétien se sent alors en danger, il réagit en cité assiégée. "Durant le XVI siècle, le seul tribunal d'inquisition de Tolède, en Espagne, a conduit 644 procès pour blasphèmes, dont 600 avec condamnations ${ }^{25}$." La législation ne s'adoucira en Europe qu'à la fin du XVIII siècle,

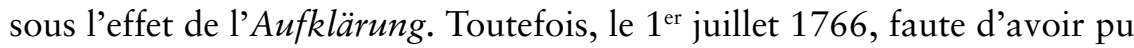
obtenir sa grâce de Louis XV, le chevalier de la Barre fut écartelé publiquement, à l'âge de dix-neuf ans, après avoir eu la langue et les poignets tranchés. Pour quels délits? Entre autres, pour blasphème (il aurait refusé de se découvrir devant une procession, et aurait détruit un crucifix, à coups d'épée) - ce fut, en Europe, sauf erreur, le dernier supplice de ce genre, et le dernier condamné à mort pour blasphème, en plein XVIII ${ }^{\mathrm{e}}$ siècle, tout de même (Gallo, 1999).

Ainsi donc, l'on a longtemps surveillé de près, en Europe, le dangereux petit membre, à savoir la langue, dont parle l'épître de saint Jacques durant tout son chapitre troisième, où il le traite de fléau $(\mathrm{Jc} 3,6)$. Le crayon, la plume et le pinceau ont été suspects de lui servir de prothèse et logés à la même enseigne. La retenue, notamment à l'égard du nom de Dieu, qui ne doit pas être invoqué en vain, fut considérée durant des siècles comme obligatoire par toutes sortes de gens, du haut en bas de l'échelle sociale. Même la familiarité dans la manière de dire Dieu pouvait être suspecte. Le 31 décembre 1823, Goethe écrit à son ami Eckermann: «les gens traitent le nom divin comme si l'Être suprême n'était guère plus que leur égal. Sinon, ils ne diraient pas: le Bon Dieu. S’ils étaient pénétrés de sa grandeur, ils en perdraient la parole et, par vénération, n'oseraient pas le nommer. " L'on rapporte aussi que lorsque l'on se moquait du Christ, Dostoïevski devenait blême et quittait les lieux.

\section{Le moment libertaire}

Dès la fin du siècle des Lumières, la raillerie atteint la figure de Dieu dans quelques caricatures de l'époque révolutionnaire (De Baecque 1988). L'une d'elles, intitulée Arrivée du pape en paradis, transporte le spectateur au ciel; Jésus est sagement assis à une table d'écolier et écrit sous la dictée de Dieu le Père; la Colombe du Saint-Esprit en perroquet est juchée sur son perchoir. Toutefois ce sont surtout les ecclésiastiques, pape en tête, qui

25. J. Delumeau, interviewé dans Le Point, n 1743, 9 février 2006, p. 49. 
constituent la cible. De Dieu, la Révolution va avoir besoin, sous la forme de «l'Être suprême» — l'allure que lui prête la gravure de J.-B. Compagnie, en 1794, est celle, traditionnelle, de Dieu le Père ${ }^{26}$. Jésus aussi est réquisitionné, dans les thèmes de prédilection que l'on devine (Jésus chassant les vendeurs du temple; Jésus docteur, lors du sermon sur la montagne); si bien que Jésus est lui aussi ménagé et mis "à l'abri de toute contamination visuellement désobligeante» (Langlois 1989, 351), sauf de manière furtive $^{27}$. Dans une "gravure à l'eau forte à la manière noire ", de 1792, Le Nouveau Calvaire ${ }^{28}$, un autre que le Christ est cloué en croix, en l'occurrence Louis XVI, figuré en martyr «crucifié entre ses deux frères, le comte d'Artois et le comte de Provence; sous l'apparence d'un moine prêcheur à cheval sur la Constitution, Robespierre lui présente l'éponge imbibée du fiel des nations régicides ", tandis que la duchesse de Polignac enlace le pied de la croix et que Marie-Antoinette, encore debout, est proche de défaillir avec ce "didactisme un peu laborieux ${ }^{29}$ ", qui numérote chaque figure et l'identifie dans la légende, un pas nouveau est franchi avec l'utilisation de l'image cardinale de l'iconographie chrétienne. Mais ce détournement de la croix, tout bien pesé, ne brocarde pas Jésus lui-même.

Sous la Restauration, l'État devra dresser contre la caricature antireligieuse un mur de surveillance. Le 8 septembre 1829 encore, le ministère de l'Intérieur diffusa une circulaire prévenant de ce qui attendait ceux qui offenseraient la religion (Jeannenay 2006). Il n'empêche. Dans le catalogue de l'exposition de la Bibliothèque Nationale de France à Paris, L'Enfer de la Bibliothèque. Éros au secret, on découvre, datant des années 1830-1840, quelques innovations symptomatiques, parmi lesquelles un Crucifix avec une femme nue à la place du Crucifié ${ }^{30}$ et aussi, dans une scène se déroulant au

26. L'invention de la lithographie par Alois Senefelder, en 1798-1799, va donner un nouvel essor à l'art de la caricature.

27. La Crucifixion est ridiculisée, à la sauvette mais nettement, dans une estampe anglaise de 1793 de James Gillray (1757-1815) mise sur le compte des Français, Louis XVI prend congé de sa femme et de sa famille (un moine tient un crucifix: le Crucifié a le corps convulsé et ouvre grand la bouche; son nez est caricatural); voir Dupuy (2008, cat. $\mathrm{n}^{\circ} 14$ et p. 31 et 114$)$.

28. Voir Baridon et Guédron $(2006,112)$. La gravure $(26,3$ x 19, 5) est attribuée à Michel Wébert. Elle est conservée à la Bibliothèque Nationale de France, cabinet des Estampes.

29. L'expression est des auteurs cités dans la note précédente, comme la partie de la description entre guillemets.

30. «Palais de l'archevêque» (cat. 144, ill. 67 e): l'étreinte du prélat et de sa courtisane a lieu sur un divan sous un crucifix féminin (paru dans Portes et fenêtres, Paris, 1835). 
musée, incidemment, un Christ en érection ${ }^{31}$. Là encore, il se pourrait que de telles initiatives, qui s'octroient le droit de tirer le motif très vénérable du Sauveur en croix du côté d'un libertinage érotique déclaré, n'aient pas eu de précédent. La France serait-elle un cas à part? Pas du tout.

En Allemagne, jusqu'à la fin du XIXe siècle, le blasphème est poursuivi, non comme crime spirituel mais comme scandale public. Il en va de même en Angleterre et en Amérique: les peines prononcées contre les blasphémateurs n'ont pas tant pour but de protéger le dogme que de sauvegarder l'ordre public, la décence, le respect des sentiments religieux de la population ${ }^{32}$.

C'est contre cette tradition du contrôle «bourgeois et clérical» des signifiants de Dieu et de la répression des «abus" que l'on s'est insurgé à partir des années 1870, en parole comme en peinture. L'on assiste alors à une véritable offensive antichrétienne. Ce fut d'abord un assaut d'idées par lesquelles rationalistes, anticléricaux et libres penseurs de tous horizons, provenant de la philosophie, mais aussi des sciences (médecine), qui s'étaient juré de venir à bout du christianisme, s'en prirent pêle-mêle aux apparitions (de Lourdes et de La Salette), à l'inhumation (au profit de la crémation), aux dogmes, etc. Ce fut une guerre d'idées et une guerre d'images. De 1870 à 1914, les journaux satiriques abondent en Europe, spécialement à Paris, capitale incontestée de la caricature entre 1870 et 1900, avec pas moins de 139 journaux spécialisés (parmi lesquels Psst! de Forain, qui prit des positions antidreyfusardes et antisémites). Quatre-vingt-quatorze autres y feront leur apparition entre 1900 et 1914 (L'Éclipse, L'Assiette au beurre $\left.{ }^{33}\right)$.

La Bible, les dix commandements (Doizy et Lalaux 2005, 63), la vie de Jésus, les dogmes, les sacrements, le catéchisme, c'est le contenu même de la foi chrétienne, et pas seulement le clergé et ses exactions réelles ou présumées, qui sont soumis à la critique, comme jamais auparavant. Le Père éternel est représenté sous les traits d'un vieux philosophe le nez chaussé de

31. Ill. 72 et cat. 147, p. 234 (paru dans Passe-temps, recueil publié soi-disant à Londres mais en fait à Paris en 1840).

32. "Blasphème", Catholicisme, col. 82.

33. L'Express, du 9 février 2006, p. 57, reproduit la couverture du n 300 du 29 décembre 1936, qui annonce clairement un «numéro anti-chrétien de Grandjouan », et où l'on voit un Jésus nu tout à fait lamentable; Le Nouvel Observateur du 9-15 février 2006, p. 58, reproduit une couverture de cette revue due à Kupka, en 1904, "qui montre, avec un talent rare, un prêtre en train de comprimer la tête d'un moribond pour lui faire cracher son or "; voir Dixmier et al. (2005). Pour d'autres caricatures, voir É. et M. Dixmier (1974). Pour l'ensemble de la presse satirique, Ducatel (1975). 
lunettes, la barbe jaunie par l'usage du tabac et de la bière, les poches bourrées d'obligations et d'actions de chemins de fer (Doizy et Lalaux 2006, 18-22). La Trinité n'est pas épargnée. Un dessin légendé «Où il y a de la gêne, il n'y a pas de plaisir » la montre à l'étroit dans l'espace d'une hostie: Dieu le Père, qui s'y est niché le premier, doit faire sa place à son Fils, qui n'y rentre que de justesse en lui enfonçant malencontreusement la traverse de la croix dans l'œil, la Colombe étant piétinée par eux deux (Doizy et Lalaux 2005, 113). La conception virginale du Christ est évidemment la cible des sarcasmes (Doizy et Lalaux 2005, 110-112 ${ }^{34}$ ). Mais pas impunément: "les directeurs du journal La Calotte (de Marseille et du Havre) passent plusieurs mois en prison pour une représentation de Marie enceinte en train de tricoter des chaussettes " (Doizy et Lalaux 2005, 40) sous l'œil perplexe de Joseph; et de proche en proche toutes les scènes de sa vie et de son ministère (voir Doizy et Lalaux 2005 : le Baptême, 112; l'Ascension, 62 et 114), y compris la Crucifixion elle-même, que les croyants sont en droit de percevoir comme le haut lieu par excellence du sacré chrétien.

L'un des premiers à franchir le seuil de «l'étape libératoire» de l'histoire de la Crucifixion est un artiste belge contemporain de Nadar et de Daumier, Félicien Rops (1833-1898; voir Rops 1998). Parmi ses œuvres les plus osées, La Tentation de saint Antoine $\left(1878^{35}\right)$. Le père des moines est à son pupitre $^{36}$ en train de méditer, comme l'indique le livre ouvert devant lui, sur la continence de Joseph poursuivi par les ardeurs de la femme de l'eunuque Potiphar, grand sommelier du pharaon (Gn 39,7-20). Sa méditation est perturbée par un cauchemar: sur la croix, à la place du crucifié ${ }^{37}$ poussé de côté par un Satan ricaneur déguisé en polichinelle et tirant la langue, une femme plantureuse, la tête ornée, en guise de couronne d'épines, d'une couronne de fleurs; les anges en pleurs de la tradition de Giotto ont été

34. «Saint Joseph était toujours représenté avec une gigantesque paire de cornes et assez souvent avec un air ahuri et benêt devant le ventre métamorphosé de son épouse » (Lalouette 1992, 55).

35. La première œuvre de Rops sur ce thème est de 1858; voir Félicien Rops. Rops suis, aultre ne veulx être, ouvrage réalisé à l'occasion des expositions commémoratives de Félicien Rops à Namur, Corbeil-Essonnes et Québec en 1998 (Bonnier et al. 2000, 3839). Sur 1878, «année-phare» pour Rops, voir p. 62.

36. Dessin aux crayons de couleur, conservé à Bruxelles (Bibliothèque Royale); voir M. Draguet (Bonnier et al. 2000, 54-55); aussi De Landsberg (2001, 143).

37. «Le principe de substitution de l'image sacrée par l'image sensuelle était déjà présent dans le célèbre ouvrage de M. G. Lewis, Le Moine [1795] et encore dans La Faute de l'Abbé Mouret de Zola... " (Védrine 2002, 294-295). 
remplacés par des putti macabres; un cochon, groin dressé, assiste à la scène; le titulus, enfin, a troqué son inscription traditionnelle, INRI ${ }^{38}$, pour une autre, qui l'est moins, EROS. Cette peinture a fait aussitôt scandale. Mais «quelques années plus tard, cette œuvre éblouira Freud qui y verra "une magnifique illustration de cas type de refoulement chez les saints et les pénitents” " (Freud 1986, 173-174). Quant à Rops, dans une lettre à un ami, il se défendra d'avoir jamais voulu attaquer la religion ${ }^{39}$. Faut-il le croire? Quoi qu'il en soit, la représentation en croix, non d'une sainte dûment vêtue, comme cela s'est produit parfois dans l'art médiéval avec sainte Kümmernis ${ }^{40}$, mais d'une femme nue à la place de Jésus, et nullement souffrante, était jusqu'alors, semble-t-il, absolument inconnue, où que ce soit ${ }^{41}$. À la même époque, qui est aussi celle de l'engouement pour la photographie, on trouve de nombreuses photos de femmes nues en croix, à des fins expérimentales sur l'esprit desquelles il est difficile d'être au clair.

Durant des siècles, il n'était venu à l'idée de personne de brocarder le motif de la Croix ou une figure de Dieu le Père. C'était impensable, ou du moins irréalisable: en tout état de cause trop dangereux. Les lois de certains États européens (Allemagne, Grande-Bretagne, Pays-Bas) prévoient encore des sanctions contre le sacrilège, et un article du code de procédure pénale d'Alsace-Lorraine, jamais appliqué mais pas encore abrogé, punit de

38. Ihesus Nazarenus Rex Judœerum (Jn 19,19).

39. «Il faut surtout que tu éloignes de la tête des gens toute idée d'attaque à la religion ou d'éroticité ", déclare Rops dans une lettre à François Taelmans (Bonnier et al. 2000, 135). Rops s'est d'ailleurs toujours défendu d'être un pornographe blasphémateur; voir, dans le même ouvrage, N. Arambasin, «Le Diable sans Dieu: l'allégorie du sacré selon Rops », p. 203-215.

40. Schmitt (1995, 268-269) évoque «sainte Wilgeforte, la sainte barbue des Flandres. Pour Schnürer et Ritz, l'étrange image de cette sainte crucifiée serait née d'une méprise: les populations du Nord n'auraient pas reconnu l'image du Christ dans le Volto Santo importé par les marchands lucquois, mais celle d'une sainte crucifiée, barbue et vêtue d'une robe" (voir aussi la suite).

41. Dans l'exposition organisée en 1997 à la Bibliothèque Nationale de Paris, sur le thème de «L'art du nu dans la photographie », l'œuvre de Rops (essentiellement La Tentation de Saint-Antoine) était confrontée à des photographies de femmes en croix. Sur ce thème, voir encore le catalogue de l'exposition Jardin de Eros, Barcelone, 1999 (p. 100, 101, 147, etc.), qu'aucun musée français n'a voulu accueillir, et Zänker (1998), qui présente également des femmes en croix ayant servi à illustrer le Marquis de Sade. Il y eut néanmoins dans l'art des siècles antérieurs des représentations de femmes en croix — mais habillées (Llompart 1973; fresque anonyme de la fin du XVI ${ }^{\mathrm{e}}$ s. ou du XVII ${ }^{\mathrm{e}}$ s.). 
trois ans de prison l'outrage à $\mathrm{Dieu}^{42}$. Cette situation est révolue depuis plus d'un siècle en "France de l'intérieur ». La séparation des Églises et de l'État, prononcée en 1905, a grandement contribué à y rendre la religion «caricaturable».

\section{Dieu dans l'art et les langages visuels au $\mathbf{x x}^{\mathrm{e}}$ siècle en Occident}

On peut parler, par la suite, d'une certaine «mort esthétique» de Dieu dans la culture et l'art, mais à condition d'introduire une distinction (Bœspflug 1991). Les thèmes de Dieu le Père ou de la Trinité s'estompent inexorablement, jusqu'à disparition quasi totale; à l'inverse, l'on constate une hypertrophie du thème de la Croix dans l'art européen de la seconde moitié du XXe siècle: témoins Bacon, Guttuso et Saura (Collectif, 2002), et déjà dans la première chez Rouault, Jawlensky et Picasso (Schmied 1980; Rombold et Schwebel 1983; Bœspflug et al. 2000).

L'histoire de Dieu, du Dieu judéo-chrétien, dans l'art européen du $\mathrm{XX}^{\mathrm{e}}$ siècle, et plus généralement dans les langages d'images (cinéma, télévision, photographies et dessins d'art et/ou de presse, publicité, dessins humoristiques, bandes dessinées, outils pédagogiques en tous genres, jeux vidéo) est encore à écrire. Nous manquons de recul pour le faire. Mais rien n'interdit de se livrer à quelques coups de sonde, par exemple dans l'art théâtral, les dessins de presse et les «unes» de magazine. Ils permettent de vérifier que la frontière entre le toléré et le non-toléré ne cesse de se déplacer.

S'agissant du théâtre, Oscar Panizza fut condamné à un an de prison ferme, en 1896, par la cour royale de Munich, en raison des insolences de son Le Concile d'Amour, publié à Munich et Zurich l'année précédente et racontant comment Dieu en son conseil projette de punir l'humanité corrompue en lui infligeant la syphilis (Panizza 198343). Près d'un demi-siècle plus tard, dans Les Verts Pâturages de Marc Connelly, Dieu reçoit les trois patriarches de l'histoire sainte juive (Abraham, Isaac, Jacob) dans son

42. R. Debray, dans Le Nouvel Observateur, 9-15 février 2006, p. 56. Le statut particulier de l'Alsace-Lorraine fait que son Code pénal conserve encore - telle une relique que d'aucuns voudraient voir abolie au plus vite — à l'article 166, un vestige de la loi allemande stipulant: "Celui qui aura causé un scandale en blasphémant publiquement contre Dieu par des propos outrageants, ou aura publiquement outragé l'un des cultes chrétiens [...] ou qui, dans une église ou un autre lieu consacré à des assemblées religieuses, aura commis des actes injurieux ou scandaleux, sera puni d'un emprisonnement de trois ans au plus.»

43. Dieu le Père apparaît à la page 60 . 
bureau: il est figuré en notaire de province. La pièce est nettement plus sage que la précédente. Il n'empêche qu'elle a encore suscité une levée de boucliers: l'honneur de Dieu, a-t-on soutenu, y était gravement offensé. Il est instructif de parcourir les articles de l'époque se prononçant avec force raisons pour ou contre, en particulier ceux des théologiens qui s'en mêlèrent: le père Roguet, dominicain, dans un article de $S e p t$, et le père Doncœur, jésuite, dans un article des Études, prirent leur plus beau stylo pour défendre la pièce contre la horde d'esprits chagrins qui ne voyaient pas d'un bon œil que l'on fît sortir Dieu de la coulisse céleste. Le second révérend fit observer au passage que l'apparition de Dieu sur scène était strictement prohibée par la loi anglaise depuis la Réforme (Bœspflug 2006b). Achevons ce survol. Un demi-siècle plus tard est porté sur les planches du Palais de Chaillot à Paris un Job (celui d'André Engel) faisant apparaître Dieu le Père: cette fois plus personne ne se demande s'il sied ou non qu'un acteur, Roger Dumas, le partenaire de Gabin dans Rue des prairies, et acteur de Fort Saganne, endosse ce rôle. Mettre Dieu en scène est en Europe (continentale, ce soit dit pour réserver le cas anglais) comme un droit acquis depuis les mystères médiévaux. Qui oserait revenir là-dessus?

Voici maintenant quelques dessins de presse. Dans les années 1980, le journal Le Monde ne se gêne pas pour faire apparaître périodiquement un dessin à mi-distance entre le genre humoristique bon enfant (comme chez Effel ou Piem) et une veine plus ou moins grinçante. Voici quelques spécimens :

1. le jour de Noël 1987 (Noël! ce qui ajoute tout de même un certain piment de provocation), année de parution de beaucoup d'éditions ou de rééditions de la Bible, Dieu le Père aussi a son cadeau, qu'il découvre avec un plaisir visible d'auteur comblé: le livre qu'il a écrit continue de se vendre à merveille (le dessin illustre l'article intitulé: «une nouvelle rage biblique»), ce qui lui vaut des droits confortables; c'est bon enfant;

2. lors du bicentenaire de la Révolution française (Le Monde, 6 janvier 1989), un dessin de Cagnat, montrant Dieu le Père consolant tant bien que mal un Louis XVI céphalophore: «te bile pas, on va se refaire» (manière de suggérer que les deux ont partie liée, et que Dieu le Père n'est pas en meilleure posture, bien qu'il porte encore la tête sur les épaules);

3. lors de la fusillade de l'Esplanade de la Mosquée à Jérusalem, un juif bien reconnaissable en chapeau, la kalachnikov encore fumante, 
quitte l'endroit, qui est jonché de cadavres de Palestiniens ensanglantés et sans vie; au ciel, se prélassant mollement sur une nuée, Dieu s'interroge non moins mollement: « je devrais peut-être faire une déclaration ». L'humour ici, est acide; c'est de la dérision dirigée à l'encontre des déclarations, mais aussi de Dieu et de sa providence.

4. «Dieu peut-il tout? », un dessin signé Serguej, illustre un article fort savant et spéculatif de Roger-Pol Droit sur un ouvrage de philosophie médiévale. Dieu le Père, nu, songeur, est assis sur le bord du lit, en situation d'impuissance, tandis que sa parèdre lui fait la tête pour cette raison. Ce type de dessin sera reçu comme scandaleux ou comme franchement rigolo, ad modum recipientis. Pour le théologien que je suis, il est bénin, voire insignifiant. Pour l'historien que j'essaie d'être aussi, il est le signe de ce qu'une limite naguère impossible à transgresser a désormais été nettement repoussée, vers beaucoup plus d'audace.

Troisième coup de sonde, en direction des «unes " de magazines de l'édition francophone ayant eu recours au nom de Dieu, soit tout seul, soit accompagné d'un motif de Dieu le Père ou du crucifié. Dans les années 1990, on peut presque tout faire sans soulever de protestation: le nom de Dieu, en France, est employé à tort et à travers, d'une manière désacralisée, jusque dans ses usages publics. L'occupant du Palais de l'Élysée est appelé «Dieu» et, de peur sans doute de passer pour une grenouille de bénitier, se laisse appeler ainsi sans broncher («Dieu peut-il rebondir?», Le Nouvel Observateur, 10-16 juin 1988). Ce n'est qu'un usage parmi d'autres qui illustrent la tolérance à la plus grande liberté de ton: "Dieu est-il misogyne?» (Le Nouvel Observateur, 5-11 novembre 1990), ou «Les frasques de Dieu» (L'événement du jeudi, du 31 décembre 1992).

La France, prompte à se scandaliser de toute trace d'agression ou d'impertinence antireligieuse dès lors qu'elle atteint le judaïsme, se pose $a$ contrario volontiers en championne de la liberté d'expression quand c'est un motif chrétien qui en fait les frais: la une de l'édition française de la revue internationale de rock, The Best, $\mathrm{n}^{\circ} 237$, d'avril 1988, avec la star "crucifiée » sur l'autel de ses fans, a été censurée partout en Europe - cela dit, les Allemands ne sont pas en reste ${ }^{44}$.

44. Voir Becker-Huberti (1999) [docteur en théologie, porte-parole de l'archevêché de Cologne depuis 1991]. Un pavé en italiques précise que l'auteur collectionne les caricatures ayant la religion pour thème. 
«Pourquoi Dieu n'aime pas les femmes », VSD, n 794, 19-25 nov. 1992 - est une couverture coupable d'une double maltraitance: du corps de la femme et de la croix du Christ, qui n'est pas assimilable à un désamour entre Dieu le Père et son Fils. Les affiches maniant les signifiants religieux, remarquons-le au passage, font souvent n'importe quoi: elles colportent d'assez grossiers contre-sens, au moment même où l'on se plaint d'une perte des clefs de connaissance de la religion... Irrespect et inculture (ou culture de l'à peu près) font bon ménage.

La une du hors-série "Spécial Amérique " de L'événement du jeudi, $n^{\circ} 724$, de septembre 1998, est une métaphore peinte sans le moindre ménagement: le président Bill Clinton, dit-elle, a été «crucifié » par l'affaire de sa liaison avec Monica Levinsky. Cette couverture n'est qu'un remake: la tête du président, un collage, remplace celle de l'acteur principal dans l'affiche du film Larry Flint, de Milos Forman (1996; voir Saint-Martin 1999), affiche qui avait provoqué une telle levée de boucliers qu'elle avait finalement été retirée.

Sur la couverture de la revue économique Bilan, $\mathrm{n}^{\circ} 186$, d'août 2005, j'ai découvert pour la première fois une image du Christ couronné d'épines et en costume-cravate. À ma connaissance, cette couverture n'a provoqué aucun drame. On imagine ce qu'aurait pu provoquer ailleurs la même, mutatis mutandis, avec le Prophète de l'islam en complet-veston!

\section{Conclusion}

L'histoire qui vient d'être retracée avec des bottes de sept lieues éveille l'impression d'une évolution linéaire, où certains verront un progrès bénéfique, d'autres une lamentable décadence, vers une permissivité de plus en plus grande des sociétés chrétiennes ou jadis telles envers les caricatures religieuses. En réalité, cette image de continuité est trompeuse, car chacune des cinq périodes distinguées commence par une percée au prix d'un passage en force par rapport auquel la suite de ladite période se situe en retrait. Nul ne saurait dire, de ce point de vue, si le début du XXI siècle ne va pas se solder par la réapparition de nouvelles interdictions - pour endiguer des vagues d'irrespect qui ne sont pas du goût de tous et préserver la paix civile des sociétés multiethniques et multi-religieuses. L'actualité, hélas, est à la construction de nouveaux murs...

Mais de fait, la situation iconique actuelle de Dieu et des «choses de la religion ", en France et en Europe, peut-être aussi au Québec, se caractérise par la récente perte d'égard contractuel, civil, pour le nom et l'image de 
Dieu — je parle toujours du Dieu chrétien, mais en songeant aussi à ce qui peut atteindre du même coup les figures fondatrices du judaïsme et de l'islam, il est vrai moins nombreuses et donc moins vulnérables. L'image religieuse, fût-ce l'image de Dieu, est considérée chez nous comme un patrimoine disponible, un fonds d'archives, sans plus. Chacun est libre d'en faire ce qu'il veut. Il arrive que les instances ecclésiales, sous la pression des protestations des fidèles, dénoncent des «abus» censés proches de l'injure et du mépris, s'élèvent contre certains bricolages d'une audace jugée excessive, voire obtiennent des pouvoirs publics que telle publicité soit interdite et tel livre retiré de la vente, tel INRI de Serge Bramly et Bettina Rheims (1998). Mais ce sont là des cas exceptionnels, et l'idée même d'un strict contrôle des signifiants visuels ou d'une "police des images" ne compte pas parmi les projets prioritaires des responsables sains d'esprit. La tentative de faire voter par le Parlement français une nouvelle loi en vue de la répression du blasphème a échoué.

Il reste vrai, néanmoins, que les deux dernières phases de l'histoire décrite, on l'oublie trop souvent, recouvrent un phénomène dont il n'est pas exagéré de dire qu'il est tout nouveau à l'échelle des siècles. Témoin l'exposition "Corpus Christi. Les représentations du Christ en photographie, 1855-2002» (Paris, Hôtel de Sully; Perez 2002), ou les actes d'un récent colloque tenu à Lausane en mai 2009 sur «Jésus en représentations. Romans, films, arts visuels au XXe siècle ». La figure de Jésus, en particulier, est tirée à hue et à dia (Bœspflug, à paraître). Une telle liberté, en toute impunité, de bricolage, de polémique, de caricature, de réquisition complaisante ou militante pour les besoins de diverses causes (féministes, laïcistes, homosexuelle, politique, anticléricale, publicitaire et marchande), aussi bien sous forme graphique que dans le registre littéraire (Sarrazin 1993; Tête 1996), eût été encore inconcevable avant la fin du XIX ${ }^{e}$ siècle.

Mais qu'en déduire? La libre manipulation des signifiants les plus respectés de la religion longtemps dominante est un phénomène récent et sans pareil, semble-t-il, dans l'histoire de l'humanité. Le mauvais usage de l'histoire consisterait à tenir la trajectoire retracée pour une loi universelle. Car il faut tenir compte impérativement du fait que la sensibilité religieuse ne se laisse pas brusquer sans réagir. On ne la fait pas évoluer par décret. Certaines formes de l'art contemporain, certaines manières de dire, d'écrire et de dessiner qui paraissent être des acquis impossibles à remettre en question dans les sociétés démocratiques, continuent de choquer certaines fractions de la population - un très remarquable dossier de la revue Kunst 
und Kirche ( «eibebaum Religion, zum Beispiel Polen», 2007, n 3) le démontre sur l'exemple de la Pologne de ces deux dernières décennies. Les manières de dire et de peindre que les Européens sont désormais entraînés à tenir pour tolérables sont jugées offensantes, voire blasphématoires à peu près partout ailleurs dans le monde. L'histoire des caricatures de Yahveh ou d'Allah, de Ganesh, de Bouddha ou de Confucius, se résumerait à fort peu de choses, sauf erreur. On ne saurait par conséquent postuler que l'évolution du monde chrétien est valable pour l'ensemble de la planète ${ }^{45}$. Au contraire, l'on fera bien de se persuader que l'Europe, surtout en sa partie occidentale, est à cet égard une aire de civilisation tout à fait à part (Goody 2003).

Ultime précision. Ce phénomène, contrairement à ce que l'on pourrait croire d'abord, est d'interprétation complexe, et pas nécessairement négatif à tous égards, y compris d'un point de vue chrétien. Et quitte à surprendre, je dirais que comme théologien, je suis prêt à soutenir, entre autres — mais il y faudrait un autre article —, qu'à la faveur du processus esquissé ici et de la mondialisation, la croix est devenue en peu de temps, sans que quiconque ait jamais songé à programmer une telle évolution — pas même la Congrégation romaine De Propaganda fidei -, l'un des symboles mondiaux, voire le paradigme par excellence de l'humanité injustement maltraitée (Bœspflug 2004). En quoi semble se vérifier ce que disent au fond tous les traités théologiques sur l'universalité des effets de la Rédemption: Deus crucifixus capax totius hominis.

\section{Références}

Baltrusaitis, J. (1981), Le Moyen Age fantastique. Antiquités et exotismes dans l'art gothique, Paris, Flammarion.

BARIDON, L. et M. GUÉDRON (2006), L'art et l'histoire de la caricature, Paris, Citadelles \& Mazenod.

BARTSCH, A. (1920-1923) [Vienne 1802-1821], Le Peintre-Graveur, 21 vol., Würzbourg.

Becker-Huberti, M. (1999), “Optisches-Juckpulver” [poil-à-gratter optique ?]: Karikatur und Kirche ", Renovatio, 55/3, p. 106-115.

45. Contrairement à ce qui est suggéré ici ou là, par exemple dans Gardes et Doizy (2008). 
Bæespflug, F. (1991), «Peut-on parler d'une mort de Dieu dans l'art? À propos d'une thèse de Wolfgang Schöne ", dans D. PaYot, dir., Mort de Dieu, fin de l'art, Paris, Cerf/Cerit, p. 15-33.

Bøespflug, F. (2004), «La crucifixion déportée. Sur la sécularisation d'un thème majeur de l'art chrétien ", dans J.-M. Husser, dir., Religions et modernité, Actes de l'université d'automne de Guebwiller (27-30 octobre 2003), CRDP de l'Académie de Versailles (Les Actes de la Desco), p. 141161. Bayard.

(2006a), Caricaturer Dieu? Pouvoirs et dangers de l'image, Paris, (2006b), «Dieu en images, Dieu en rôles. Positions théoriques et faits d'histoire ", dans J.-P. BORDIER, A. LASCOMBES, dir., Dieu et les dieux dans le théâtre de la Renaissance, Actes $d u X L V{ }^{e}$ Colloque International d'Études Humanistes (Tours, Centre d'Études Supérieures de la Renaissance, juillet 2002), Turnhout, Brepols, p. 11-35.

(2008) «Caricaturer Dieu? Se moquer du Prophète? Liberté d'expression et respect du sacré ", Bulletin du Centre Protestant d'Études, 60/1-2, p. 25-47.

(à paraître), "Jésus à hue et à dia. Sur les divers usages du motif du crucifié dans les langages d'images du Xx ${ }^{e}$ siècle», dans Ph. KAENEL et P. GISEL, dir., Jésus en représentations.

Bespflug, F. et al. (2000), Le Christ dans l'art, Paris, Bayard.

Bonnier, B., V. Leblanc, D. Prioul et H. Védrine, dir. (2000), Félicien Rops. Rops suis, aultre ne veulx être, Paris, Éditions Complexe.

Bramly, S. et B. Rheims (1998), I.N.R.I., Paris, Albin Michel.

CAmille, M. (1992), Image on the Edge. The Margins of Medieval Art, Londres, Reaktion Books.

CARofF, F. (2006), "Portrait des musulmans dans les enluminures ", dans M. ArKoun, dir., Histoire de l'Islam et des musulmans en France du Moyen Âge à nos jours, Paris, p. 178-191.

Casagrande, C. et S. Vecchio (1991), Les Péchés de la langue. Discipline et éthique de la parole dans la culture médiévale, Paris, Cerf.

CelSE (1999), Contre les chrétiens, prés. et trad. par L. Rougier, Paris, Phébus.

Charbonneau-Lassay, L. (2006), La mystérieuse emblématique de JésusChrist. Le Bestiaire du Christ. Mille cent cinquante-sept figures gravées sur bois par l'auteur, rééd. Paris, Albin Michel. 
Christin, O. (1991), Une révolution symbolique: l'iconoclasme huguenot et la reconstruction catholique, Paris, Éd. de Minuit, p. 159-174.

(1994), "Sur la condamnation du blasphème (XVI ${ }^{e}-X V I I^{e}$ siècle) ", Revue de l'Histoire de l'Église de France, vol. 80, n 204, p. 43-64.

Clair, J. (2005), Une leçon d'abîme. Neuf approches de Picasso, Paris, Gallimard.

Collectif (2002), Antonio Saura, Crucifixions/Crucifixiones, cat. d'expo, Musée d'art moderne et contemporain de Strasbourg.

Collectif (2004), Gravures du temps de la Réforme. Collection du Schlossmuseum de Gotha, cat. d'expo, Montbéliard.

Collectif (2004), Hommeanimal, histoire d'un face à face, cat. d'expo, Strasbourg.

Dandrey, P. (1997), L'Éloge paradoxal, de l'Antiquité à Molière, Paris, PUF.

De BAeCque, A. (1988), La Caricature révolutionnaire, Paris, CNRS.

De LANDSBerg, J. (2001), L'Art en croix. Le thème de la crucifixion dans l'histoire de l'art, Tournai, La Renaissance du livre (Références).

Delaurenti, B. (2007), La puissance des mots, "virtus verborum». Débats doctrinaux sur le pouvoir des incantations au Moyen Âge, Paris, Cerf.

Delumeau, J. (1981), "Morale et religion saisies à travers les farces, les sotties et les sermons joyeux", Un chemin d'histoire. Chrétienté et christianisation, Paris, Fayard, p. 30-43.

Devisse, J., M. Mollat et J.-M. Courtès (1979), L'Image du Noir dans l'art occidental, t. 2, Paris, Gallimard.

Dinkler, E. (1967), Signum crucis. Aufsätze zum Neuen Testament und zur christlichen Archäologie, Tübingen, Mohr, p. 150-153.

Dinzelbacher, P. (1999), "Étude sur l'incroyance à l'époque de la foi ", Revue des Sciences religieuses, 73/1, p. 42-79.

DiXMIER, É. et M. (1974), L’Assiette au Beurre, revue satirique illustrée, Paris.

Dixmier, M., J. Lalouette et D. Pasamonik (2005), La République et l'Église: images d'une querelle, Paris, Éditions de la Martinière.

DoIzy, G et J.-B. LaLAuX (2005), À bas la calotte! La caricature anticléricale et la Séparation des Églises et de l'État, Paris, Éditions Alternatives.

(2006), Et Dieu créa le rire. Satires et caricatures de la Bible, Paris, Éditions Alternatives. 
Ducatel, P. (1975), L'Histoire de la Troisième République vue à travers l'imagerie populaire et la presse satirique, Paris, J. Grassin.

Dupuy, P. (2008), Caricatures anglaises (1789-1815). Collections du musée Carnavalet, Paris, Nicolas Chaudun.

FreUd, S. (1971) [1949], «Étude sur la Gradiva de Jensen (1907)», Le Délire et les rêves dans la Gradiva de W. Jensen, Paris, Gallimard (Folio-essais).

Gallo, M. (1999), Que passe la justice du roi, Paris, Robert Laffont.

Gardes, J.-C. et G. DoIzy (2008), dir., Caricature et religion(s), Ridiculosa, 15.

Gauvard, C., A. De Libéra et M. ZINK (2002), Dictionnaire du Moyen Âge (DMA), Paris, Quabrige/PUF.

Goody, J. (2003 [anglais 1997]), La peur des représentations. L'ambivalence à l'égard des images, du théâtre, de la fiction, des reliques et de la sexualité, tr. de l'anglais par P.-E. Dauzat, Paris, La Découverte.

HeERs, J. (1983), Fêtes des fous et carnavals, Paris, Fayard.

HeID, S. (2006), «Kreuz», dans E. Dassmann, dir., Reallexikon für Antike und Christentum, t. 21, Stuttgart, Anton Hiersemann.

Horowitz, J. et S. Menache (1994), L'Humour en chaire. Le rire dans l'Église médiévale, Genève, Labor et Fides.

JEANNENAY, J.-N. (2006), "Quand le sacrilège était puni de mort en France ", L'Histoire, juin, p. 68-72.

Julien (2003), Misopogon, texte établi et trad. par C. Lacombrade, intro. et notes par A. de Saint-Loup, Paris, Les belles lettres (Classiques en poche; 63).

LABORDE, A. Comte de (1912-1927), La Bible moralisée illustrée conservée à Oxford, Paris et Londres. Reproduction intégrale du manuscrit du $\mathrm{XIII}{ }^{\mathrm{e}}$ siècle accompagnée de planches tirées des bibles similaires et d'une notice, 4 vol. de planches et 1 vol. de textes, Paris.

LALOUETTE, J. (1992), «Iconoclastie et caricature dans le combat librepenseur et anticlérical (1879-1914) ", dans S. Michaud, J.-Y. MOLLIER et N. SAVY, dir., Usages de l'image au XIX ${ }^{\mathrm{e}}$ siècle, Paris, Créaphis, p. 51-61.

LANGLOIS, C. (1989), "Venez voir la religion de nos pères et mères" : changement d'images, images du changement ", dans Populations et cultures, Mélanges François Lebrun, Rennes, Éd. universitaires de Rennes, p. 349357. 
LECLERCQ, H. (1907), «Âne, 1/ La calomnie de l'onolâtrie », DACL, t. 1/2, 1907, col. 2041-2042.

Lehmann, P. (1963), Die Parodie im Mittelalter, $2^{\mathrm{e}}$ éd., Stuttgart, Hiersemann.

LlOMpart, G. (1973), «El fresco de la religiosa crucificada de las Descalzas Reales de Madrid», Traza y Baza, 3, p. 53-60.

Meiss, M. (1969), French Painting in the Time of Jean de Berry. The Late Fourteenth Century and the Patronage of the Duke, Londres, Phaidon.

Mellinkoff, R. (1993), Outcasts. Signs of Otherness in Northern European Art of the Late Middle Ages, Berkeley/Los Angeles/Oxford, University of California Press.

(2004), Averting Demons. The Protective Power of Medieval Visual Motifs and Themes, 2 t., Los Angeles, Ruth Mellinkoff Publications.

MinoIs, G. (1998), Histoire de l'athéisme des incroyants dans le monde occidental des origines à nos jours, Paris, Fayard.

Molanus, Traité des saintes images, éd. F. Bœspflug et al., Paris, Cerf, 1996, t. 1.

Muller, F. (1997), Heinrich Vogtherr l'Ancien. Un artiste entre Renaissance et Réforme, Wiesbaden, Harrassowitz.

(2000), «Le thème du Pape-Antéchrist dans la polémique visuelle anticatholique dans le Rhin supérieur au XVI siècle", Simpliciana. Schriften der Grimmelshausen-Geselleschaft, 22, p. 75-90.

Panizza, O. (1983), Le Concile d'amour, tragédie céleste, trad. par J. Bréjoux, prés. par J.-M. Palmier, Grenoble, Presses universitaires de Grenoble.

Perez, N. N. (2002), Corpus Christi. Les représentations du Christ en photographie, 1855-2002, cat. d'expo, Jérusalem/Paris.

Quignard, M.-F. et R.-J. SECKel, dir. (2007), L'Enfer de la Bibliothèque. Éros au secret, cat. d'expo., Paris, Bibliothèque Nationale de France.

RANDALL, L. (1966), Images of the Margins of Gothic Manuscripts, Berkeley, University of California Press.

Rombold, G. et H. Schwebel (1983), Christus in der Kunst des 20. Jahrbunderts, Fribourg/Bâle/Vienne, Herder.

Rops, P. (1998) [1892¹], Mémoire pour nuire à l'histoire artistique de mon temps, et autres feuilles volantes, Bruxelles, Labor. 
Roy, B. (1992), Une Culture de l'équivoque, Montréal/Paris, Bellarmin/Vrin.

SAINT-Martin, I. (1999), "À propos d'un transfert d'image, le "Crucifié" entre Ève et Marie», Xoana. Images et sciences sociales, $n^{\circ}$ 6, p. 91-94.

SANSY, D. (1994), L'Image du juif en France du Nord et en Angleterre du XIIe au XVe siècle, thèse doctorale, Paris X-Nanterre.

Sarrazin, B. (1993), La Bible parodiée. Paraphrases et parodies, Paris, Cerf.

SCHMied, W., dir. (1980), Zeichen des Glaubens, Geist der Avantgarde. Religiöse Tendenzen in der Kunst des 20. Jahrhunderts, Stuttgart.

Schmitт, J.-C. (1995), «Cendrillon crucifiée. À propos du Volto santo de Lucques ", dans COLLECTIF (1995), Miracles, prodiges et merveilles au Moyen Âge. XXV Congrès de la Société des historiens médiévistes de l'enseignement supérieur public (Orléans, juin 1994), Paris, Publications de la Sorbonne, p. 241-269.

SifaOUI, M. (2006), L'Affaire des caricatures. Dessins et manipulations, Paris, Éditions Privé.

SKUBISZEWSKI, P. (2002), La croix dans le premier art chrétien, Paris, Librairie orientaliste Paul Geuthner.

TÊTE, A. (1997), Contre Dieu. Court traité du blasphème, Paris, Phébus.

VAISSE, P. (1974), Grünewald, Tout l'œuvre peint, Paris, Flammarion.

VÉDrine, H. (2002), De l'encre dans l'acide. L'œuvre gravé de Félicien Rops et la littérature de la décadence, Paris, Champion (Romantisme et modernités; 59).

Webster, R. (1990), A Brief History of Blasphemy. Liberalism, Censorship and "The Satanic Verses", Southwold, The Orwell Press.

WiRTH, J. (2000), "Les singes dans les marges à drôleries des manuscrits gothiques ", Micrologus VIII (Il mondo animale), p. 429-444.

Wirth, J., avec la coll. d'I. Engammare (2008), Les Marges à drôleries des manuscrits gothiques (1250-1350), Genève, Librairie Droz.

ZanetTi, U. (1991), «Les icônes chez les théologiens de l'Église copte », Le Monde Copte, $\mathrm{n}^{\circ}$ 19, p. 77-91.

ZänKeR, J. (1998), Crucifixae. Frauen am Kreuz, Berlin, Mann. 


\section{Résumé}

L'affaire des caricatures de Mahomet (février 2006) a mis en lumière l'existence de seuils de tolérance à la moquerie visant les choses religieuses. Ces seuils varient beaucoup d'une religion à une autre. L'auteur entend souligner à quel point ils évoluent aussi à l'intérieur de la religion chrétienne, réputée plus placide que d'autres à cet égard. Avec une méthodologie d'historien et des repères de théologiens, il retrace les cinq grandes étapes (ou situations typiques) d'une histoire spécifique, celle des rapports entre le christianisme et la dérision, notamment sous la forme graphique de la caricature des figures caractéristiques de cette religion: l'Antiquité, le Moyen Âge, la Réforme, les dernières décennies du XIXe siècle, puis la seconde moitié du $\mathrm{XX}^{\mathrm{e}}$ siècle. Il ressort que les seuils de tolérance ne sont jamais fixés une fois pour toutes et surtout varient selon qu'il s'agit de Dieu (du Christ) et de la Vierge, ou bien des saints, des curés ou des pratiques religieuses. En tout état de cause, ils sont toujours l'objet de subtiles négociations et font partie intégrante du pacte social dont chaque société a besoin pour éviter les heurts qui peuvent résulter du choc des sensibilités.

\section{Abstract}

The case raised by Muhammad's caricatures (feb. 2006) has shown that there could be limits to the mocking of religious matters. If the limits can vary from a religion to an other, in this paper it will be pointed how they evolved within the Catholic religion, that is considered more tolerant in these matters. Using a historical methodology and theological coordinates, five important landmarks or typical situations are presented within the history of the relationships between Christianity and mockery, focusing on the pictorial caricature of this religion's great figures: the Antiquity, the Middle Ages, the Reformation, the last decades of the $19^{\text {th }}$ century, and the second half of the $20^{\text {th }}$ century. It is shown that tolerance towards mockery varies depending on the "target": God, Christ, the Virgin Mary, or the saints, the priests and the religious practices. In any case, tolerance always has to be negotiated within the social pact that is central to every society, in order to prevent the problems that can result from clashes of sensitivity. 